\title{
Modelo basado en lógica difusa para la construcción de condiciones de alta calidad en el sistema educativo*
}

\author{
Fuzzy logic-based model for the construction of high quality \\ conditions in the education system.
}

Citar como: Castellanos Contreras, J. U., Pareja Figueredo, C. F. y Gutiérrez Martínez, L. C. (2020). Modelo basado en lógica difusa para la construcción de condiciones de alta calidad en el sistema educativo. CITAS, 6(1). https://doi.org/10.1 $5332 / 24224529.6360$

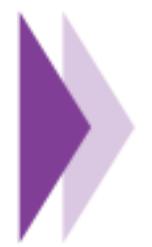

José Ulises Castellanos Contreras ${ }^{1}$; Carlos Francisco Pareja Figueredo ${ }^{2}$; Luis Carlos Gutiérrez Martínez ${ }^{3}$

1 Universidad Cooperativa de Colombia

2 Fundación de Educación Superior San José

3 Fundación de Educación Superior San José

Correo electrónico: jose.castellanos@campusucc.edu.co carlos.pareja@usanjose.edu.co

luis.gutierrez@usanjose.edu.co 


\section{Resumen}

Las bases conceptuales de la inteligencia artificial, los sistemas expertos e ingeniería del conocimiento, y la gestión son punto fundamental en la transformación digital de la educación. De esto, la lógica difusa, como sistema experto, es el pilar de nuestro modelo basado en matrices que se autorrellenan a partir de perspectivas de expertos y de directrices del Ministerio de Educación en sus matrices (sistemas cualitativos). Esto permite emigrar a datos cuantitativos a partir de reglas de inferencia (sistemas cuantitativos). Este modelo digitaliza las mallas curriculares y la gestión institucional, es decir, conocimientos y datos. Esta arquitectura digital se soporta en los sistemas de financiación universitaria, la investigación, el espíritu empresarial, el talento digital, la proyección social, la docencia, los estudiantes, entre otros. Todos estos elementos generan las matrices objetivo, las cuales proporcionan datos que soportan los elementos de alta calidad que exige el Ministerio de Educación.

Palabras clave: transformación digital, lógica difusa, reglas de inferencia, matriz objetivo, educación.

La transformación digital es un proceso que se lleva de diferentes formas en las organizaciones que aplican la Cuarta Revolución Industrial en sus actividades. Esto implica el uso de las TIC con sus elementos (cognimática, informática, telecomunicaciones, internet, redes, nanotecnología, microelectrónica, informática e inteligencia artificial, entre otros), en pro de mejorar los procesos. En el caso de las instituciones de educación superior, la transformación digital es el siguiente paso en la evolución del emprendimiento educacional, influenciado por la digitalización de la sociedad, con estudiantes que crecieron con el internet, interactuando en redes sociales a través de dispositivos móviles y manteniendo un uso intensivo de la tecnología como parte importante para su futuro profesional (Global Millennial Survey, 2014).

Esta investigación plantea que si los sistemas expertos (lógica difusa) utilizan herramientas como la transformación digital pueden autorregularse mediante sistemas de información inteligente, ya que todo está basado en la virtualidad. A continuación, se presenta un modelo fundamentado en inteligencia computacional para la gestión inteligente de la información, que integra varias arquitecturas híbridas en un sistema multinivel

\section{Abstract}

The conceptual bases of artificial intelligence, expert systems and knowledge engineering, and management are a fundamental point in the digital transformation of education. From this, fuzzy logic, as an expert system, is the pillar of our model based on matrices that are self\#filled from the perspectives of experts and the guidelines of the Ministry of Education arranged in their matrices (qualitative systems). This allows migrating to quantitative data from rules of inference (quantitative systems). This model digitizes the curriculum framework and institutional management, that is, knowledge and data. This digital architecture is supported by university financing systems, research, entrepreneurship, digital talent, social projection, teaching, students, among others. All these elements generate the target matrices, which generate data that support the high\#quality elements required by the Ministry of Education.

Keywords: digital transformation, fuzzy logic, rules of inference, objectives matrix, education.

y muestra resultados con métricas de alta calidad, indicadores y valores de gestión, posible deserción, respuestas y planes. Todo esto puede influir en la toma de decisiones, permanencia estudiantil, conocimiento requerido por el entorno, innovaciones pedagógicas, planes inferidos, políticas y objetivos de mejora continua para el fortalecimiento de la transformación digital universitaria. El artículo presenta una transición entre lo teórico y el desarrollo de software hacia la parte experimental, pero sin datos concluyentes.

\section{Lógica difusa en la transformación digital}

La lógica difusa es un método de procesamiento que se asemeja al razonamiento humano, que difiere de la lógica matemática tradicional que un ordenador puede comprender (0.1). Esta toma la lógica del razonamiento incompleto con base en la teoría de conjuntos difusos que, en su universo, presenta un abanico de posibilidades entre el sí y no (0.1). En este conjunto de universo $\mathbf{S}$, a cada elemento $\mathbf{X}$ se le asigna un valor entre 0 y 1 (ejemplo 0.5), definido por expertos, como muestra la figura 1 . 
Figura 1. Conjuntos difusos y función de pertenencia

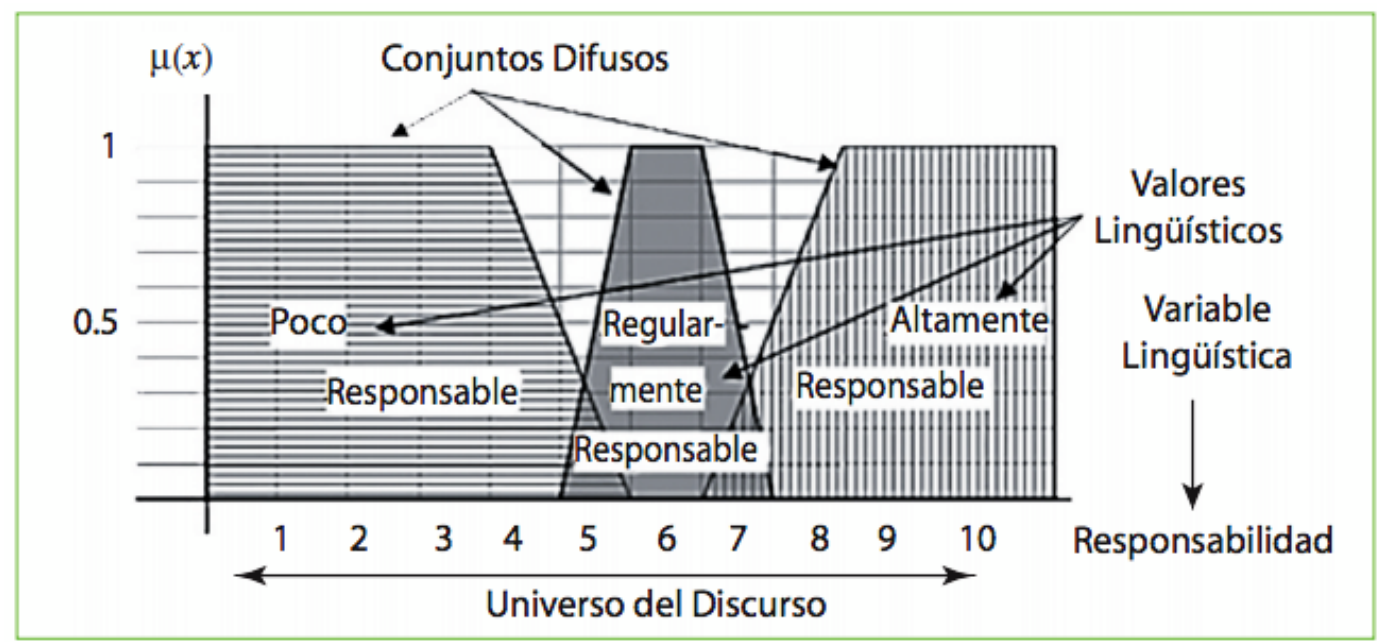

Fuente: Shinghal (2013).

En conjuntos matriciales el eje de filas representa el universo de discurso, el cual, a través de reglas de inferencia, se asigna un valor difuso a una preposición cualitativa (fuzzificación). El eje de columnas representa los grados de pertinencia asociados por reglas de inferencia, el cual le asigna un valor cuantitativo al conjunto difuso antes obtenido (desfuzzificación). Para ello los métodos más utilizados son el método del máximo, método de la media de los máximos y método del centro del área o promedio ponderación (Mendoza et ál., 2016).

El método aplicado en esta investigación fue el método máximo, que tomael vector de ponderaciones de los criterios (ai,j) con la matriz degradaciones de pertenencia (nj), usando la siguiente ecuación 1:

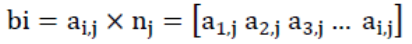

$$
\begin{aligned}
& \times\left[\begin{array}{lllllllllll}
\mathrm{n}_{11} & \mathrm{n}_{12} & \cdots & \mathrm{n}_{21} & \mathrm{n}_{\mathrm{j} 22} & \cdots & \vdots & \ldots & \mathrm{n}_{1 \mathrm{k}} & \mathrm{n}_{2 \mathrm{k}}
\end{array}\right. \\
& \left.\begin{array}{llll}
n_{\mathrm{j} 1} & \mathrm{n}_{\mathrm{j} 1} & \ldots & \mathrm{n}_{\mathrm{jk}}
\end{array}\right]
\end{aligned}
$$

Donde cada bi emplea los operadores de producto difuso algebraico y suma difusa acotada. De acuerdo con la clasificación difusa, significa que la calificación definitiva para cada elemento es $\mathrm{b}=\max (\mathbf{b} 1, \mathbf{b 2}, \mathbf{b 3}, \ldots \mathbf{b i})$, siendo max el operador que devuelve el valor máximo de varios valores difusos. Obtener este valor "b" es lo que se denomina defusificación, que no es otra cosa que transformar un valor difuso en su correspondiente valor nítido (Ma y Zhou, 2000).

\section{Metodología de implementación del modelo}

Para el desarrollo del modelo se escogió la lógica difusa de otro tipo de sistemas expertos, ya que puede diseñar un modelo sin necesidad de una base matemática inicial, y su principal herramienta está basada en reglas sin necesidad de que el usuario tenga experticia en estas, de tal forma que se puede implementar en expertos evaluadores de otras disciplinas, siendo lo ideal su aporte a un solo objetivo. El modelo implementado realiza los cálculos que promedian las calificaciones para dar un valor único de relación entre un objetivo y un producto, en los mismos términos linguiísticos, que corresponde a cada celda de la matriz ( $\mu \mathrm{P}: \mathrm{ai}, \mathrm{j})$. La matriz A conforma la base de conocimientos fuzzy con relación al producto (filas):

$\mathrm{i}=1,2, \ldots$ (cantidad de ítems de productos asociados a diferentes proyectos)

$\mathrm{j}=1,2, \ldots$ (cantidad de proyectos),

Estableciendo la matriz a partir de la ecuación 2.

$$
A: \sum_{i} a_{i, j} \times n_{j}=b_{i}
$$

Donde:

bi: es la producción de la i\#esima fila expresada de cada ítem del modelo.

nj: métrica fuzzy. Este factor se calcula según la ecuación 3.

$$
\mathrm{w}_{\mathrm{j}} \times \mathrm{r}_{\mathrm{j}}=\mathrm{n}_{\mathrm{i}}
$$

Donde:

wj: es el factor de ajuste en función de la dificultad para relacionar entre los productos y el objetivo por cada ítem. 
rj: ajuste de normalización.

Los objetivos, productos, proyectos y su análisis fuzzy se materializan en un proceso progresivo de acomodación en forma de adaptaciones y representaciones con las características flexibles en una hoja de cálculo. Mediante distintas hojas agrupadas en libros se logran las distintas partes del sistema; diferentes libros permiten hacer especificaciones para casos (figura 2).

Figura 2. Matriz de pertinencia (fuzzy) a partir de expertos, políticas

internas de la organización (caso Fundación de Educación San José)

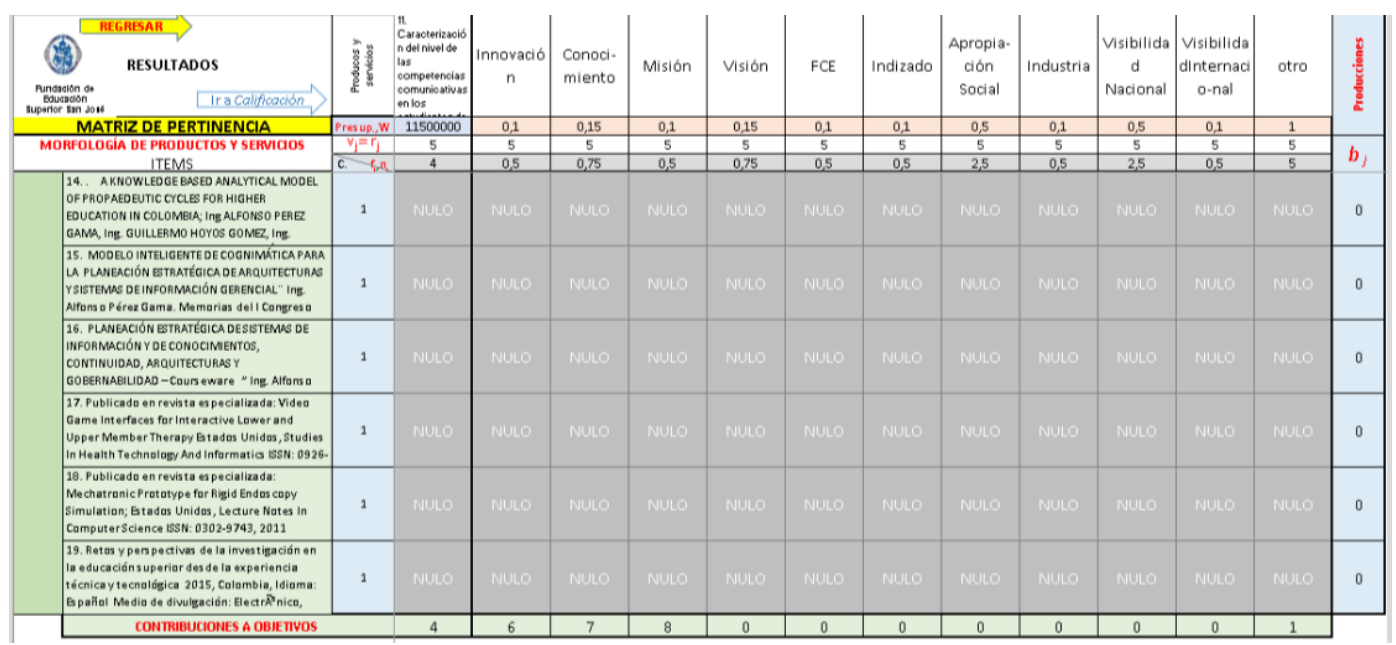

Fuente: elaboración propia.

Este mismo procedimiento se aplica para cada caso de pertinencia, donde los expertos establecen los objetivos. Otra matriz planteada es la interacción del conjunto de competencias, resultados del plan curricular (salidas) frente a los conjuntos unitarios de conocimientos donde se hacen explicitas las microcompetencias (entradas)

La interrelación se califica con valores cualitativos como alta, buena, media, regular o nula. La herramienta inteligente posibilita la intervención automatizada de varios expertos y su registro a través de la arquitectura matricial que se va completando en un plano de $360^{\circ}$. Esto posibilita la optimización, vía análisis cuantitativos, mediante el proceso de desfuzzificacio\#n. En este caso ponderamos con los créditos de cada asignatura j, nj. Para ello se elabora una matriz A de MxN, donde $\mathrm{M}$ filas representan los niveles de las competencias objetivo a obtener de la arquitectura. $\mathrm{N}$ columnas representan las áreas unitarias y funcionales de unidades de conocimientos (asignaturas y microcompetencias ofrecidas). Cada coeficiente (celda) es $\mu \mathrm{P}:$ ai, $\mathrm{j}=>\{0.1\}$, para el caso de una matriz binaria. En este caso es 1 si existe implicación directa entre el área de experticia y cada asignatura del plan de estudios del ciclo, de lo contrario es 0 .

Por otra parte, cada coeficiente representa la cohesión y coherencia de cada objetivo con la producción, que pueden contener otros valores cualitativos (lógica fuzzy) más amplios, de tal forma que aumente el grado de posibilidades (ai, $j=\{$ nulo, bajo, medio, alto\}; representado por colores: blanco, verde, rojo, azul). Para cada celda, varios expertos independientes deben dar su opinión de grado de incidencia, el cual representa la coherencia y cohesión de cada competencia con la microcompetencia de la asignatura.

El sistema promedio da resultados en los mismos términos. Estos se despliegan en una matriz de resultados que contiene la cuantificación curricular de contribución cognitiva, matriz resultante que se observa en la figura 3. 
Figura 3. Matriz de pertinencia (fuzzy) a partir de expertos, competencias y microcurrículos (caso Fundación de Educación San José)

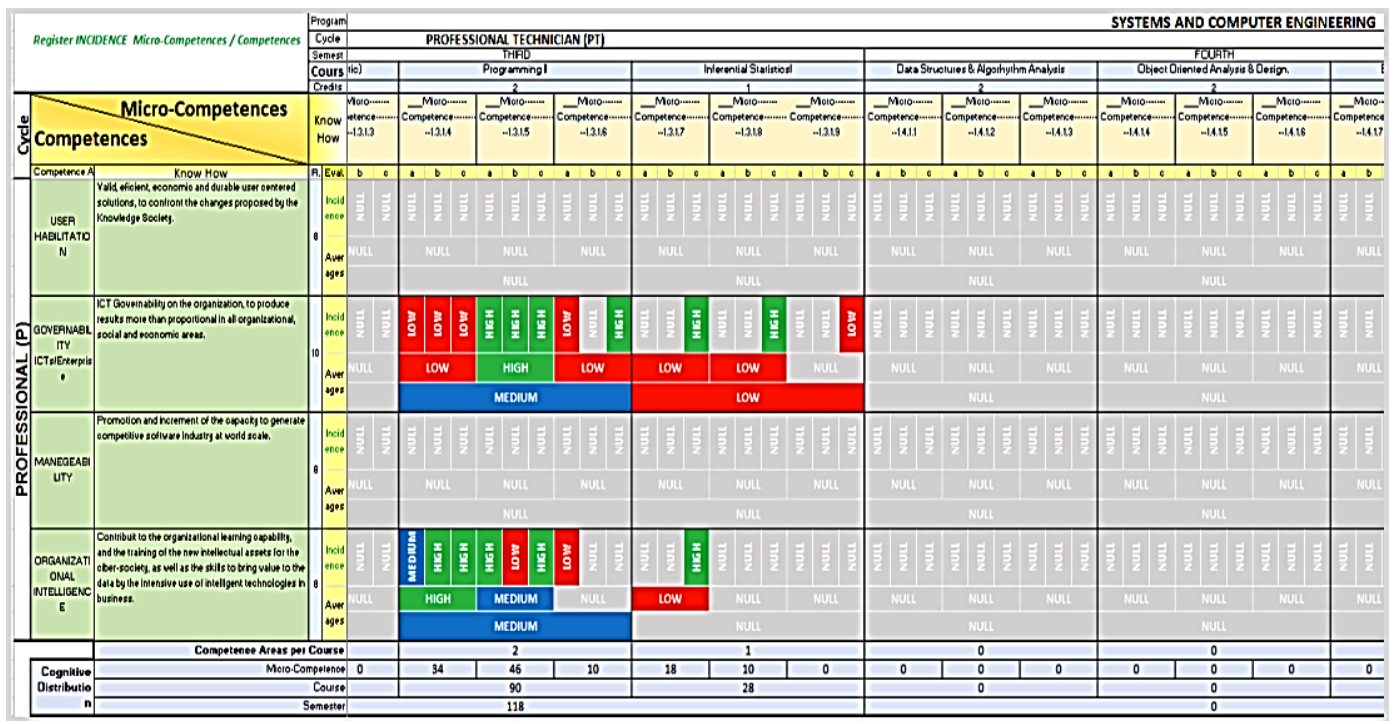

Fuente: elaboración propia.

Para el desarrollo de la matriz de la figura 3, los expertos en su análisis de coherencia curricular y cuantificación en ciclos propedéuticos (técnico profesional, tecnología y profesional) deben tener en cuenta los instructivos decretados por el Ministerio de Educación Nacional (MEN) para un plan de estudios. Estos se basan en tres componentes: modelo estructural o matriz para el análisis de coherencia curricular, gestión del conocimiento y la productividad modelo del estudiante. Esto hace que el análisis curricular posible sea en el dominio del tiempo (periodos) y en el dominio del conocimiento (por áreas y sujetos) (Ministerio de Educación Nacional, 2018).

Las siguientes matrices que se desarrollan en el modelo las establece el MEN a través de expertos nacionales, quienes definen los propósitos y objetivos de alta calidad en gestión educativa. Para ello, el Ministerio establece 14 condiciones (matrices) de calidad de carácter institucional que se deben cumplir con sus objetivos para cada matriz, dando ejemplos de verificación de cumplimiento. El modelo fuzzifica, a través de reglas de inferencia propuestas por expertos, los propósitos de cumplimiento de cada una de las matrices, haciendo posible la operación cualitativa entre estas, para luego desfuzzificarla dando valores cuantitativos, de tal forma que se pueda interpretar cuál es la variable a modificar para obtener el resultado óptimo. El modelo comienza a ser complejo en el momento en que las matrices empiezan a interactuar tanto exteriormente, cuando las variables son independientes, como interiormente cuando comparten variables. En la figura 4 se observa el diagrama de proceso de interacción de las matrices del MEN (matrices ya definidas por el MEN y que se distribuyen en sus documentos), que soportan la arquitectura de la transformación digital de la gestión educativa. 
Figura 4. Arquitectura sostenible de la transformación digital basado

en las matrices del MEN (caso Fundación de Educación San José)

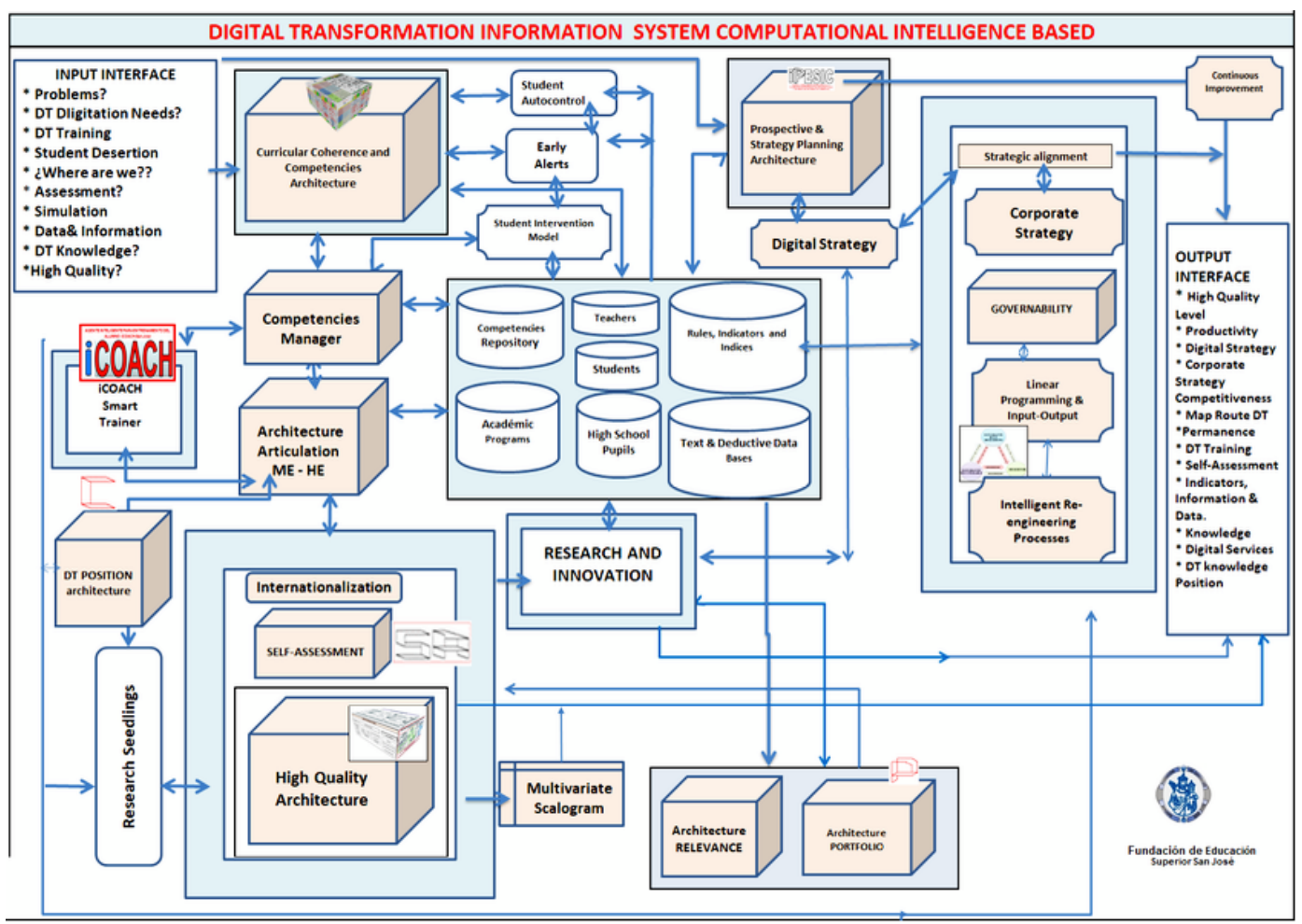

Fuente: Fundación de Educación Superior San José (2019).

En la figura 4 se observa la distribución entre grupos predefinidos por las matrices del MEN; internacionalización, investigación e innovación, administración; y cómo la arquitectura digital relaciona los subsistemas para obtener mejores y nuevas funcionalidades basadas en el conocimiento a través de un comportamiento inteligente y así ser más eficiente. El enfoque de la arquitectura de múltiples capas se podría denominar sistema de sistemas, que garantiza el cumplimiento de las políticas gubernamentales, normas y estándares, en una institución altamente compleja.

\section{Aplicaciones de la lógica difusa en investigaciones sobre la administración educativa}

El Instituto Tecnológico de Morelos (México) ha desarrollado investigaciones utilizando sistemas expertos, capaces de orientar al alumno en la elección de una carrera profesional con base en herramientas tipo test e historial del interesado. Los resultados han replanteado las técnicas de orientación vocacional implementadas y el interés desde el ámbito de la pedagogía universitaria orientada a los institutos tecnológicos. A partir de estos estudios se realizaron diferentes investigaciones que se denominaron relación entre educación y lógica difusa. En el caso mexicano, se creó una herramienta de software que es capaz de orientar al alumno en la elección de una carrera profesional dentro del sistema nacional mexicano de institutos tecnológicos (Ballester Brage y Colom Cañellas, 2006).

Con respecto a la educación virtual, la Universidad Tecnológica de Bolívar y la Universidad de Cartagena, desarrollaron una metodología para clasificar y predecir usuarios en ambientes virtuales, relacionando la interacción de los estudiantes con la plataforma y su desempeño en los exámenes. En la investigación se utilizó las herramientas de aprendizaje automático, clusterización, lógica difusa, y el algoritmo de vecindades cercanas. Los resultados muestran cómo en el tiempo un estudiante que permanece en la plataforma puede o no estar clasificado en grupos de alto conocimiento, e identificar aplicando la metodología Fuzzy K\#means para predecir el nivel de conocimiento aportado por la plataforma (De la Hoz, De la Hoz y Fontalvo, 2019).

\section{Resultados}

El avance del proceso investigativo se centra en la relación de las matrices altamente correlacionadas unas con otras, en donde dependen de la elaboración de la escala difusa y de la ponderación difusa en los objetivos implementados. Por otra parte, se ratifica que la fase cualitativa ya culminó gracias a los aportes de diferentes entes universitarios y del Ministerio de Educación que definieron las matrices a tratar. En la parte cuantitativa hay que implementar otras técnicas de tratamiento de datos para evolucionar el concepto 
del experto y así poder obtener información que retroalimente las variables.

Los resultados obtenidos en relación con estudiantes nos muestran que no existen políticas específicas o técnicas que permitan evaluar el componente motivacional. La transformación digital realiza una eficiente gestión del tratamiento de datos, pero un elemento emocional sigue siendo una variable difícil de digitalizar; esto influye al momento de evaluarla, ya que no se registra ninguna evidencia relacionada.

Los expertos hacen referencia principalmente a la elaboración de portafolios estudiantiles, visitas técnicas a empresas del sector productivo local y nacional, proyectos comunitarios en equipo, desarrollo de talleres y realización de prácticas de laboratorio, con el propósito de generar posibles variables que alimenten el modelo basado en lógica difusa, aunque la variable denominada carga emocional, debido a la virtualización, seguirá en estudio (García Ferrer, 2017). Esto influye en la toma de decisiones para la construcción de un conocimiento significativo, si se implementan estrategias constructivistas de aprendizaje por descubrimiento y resolución de problemas o la estrategia de aprendizaje asistido.

La fase cuantitativa ha logrado como resultado la ponderación de las actitudes a evaluar, la matriz resultante de gradaciones de pertenencia (nj). Estos instrumentos son indispensables para el desarrollo del sistema difuso de evaluación que se pretende como propósito final de la investigación, ya que para el diseño de las reglas difusas de inferencia se requiere la aplicación de estos. Aplicar la lógica difusa en la elaboración de los instrumentos eliminó el debate, en este caso, entre profesores, directivos y administrativos, pues el acuerdo se logra a través de los procesos difusos de expertos que solo se evidencia cuando expresan su opinión a través de cuestionarios individuales.

Este modelo constituye una valiosa alternativa en los procesos investigativos, ya que agrega objetividad al análisis de datos.

\section{Conclusiones}

La investigación presenta avances en el sector educativo, ya que se basa en técnicas de inteligencia artificial, en este caso lógica difusa, para la gestión de la información. La valoración de objetivos linguísticos por docentes y estudiantes de la lógica difusa permitió el diseño de instrumentos para su evaluación, como son las matrices de ponderación.

Uno de los grandes aportes de esta investigación es la digitalización de los conceptos de los expertos que estaban escritos en las matrices del MEN, como variables de entrada utilizables en diferentes modelos como instrumentos. Estos son indispensables para el desarrollo del sistema difuso de evaluación, gestión y desarrollo de documentos maestros, y para que el diseño de las reglas difusas de inferencia sea aplicado en estos.

La aplicación del modelo ha tenido inicialmente un rechazo por la comunidad educativa, principalmente por los docentes y administrativos, debido al desconocimiento de este tipo de modelos y a los resultados a largo plazo, debido a la no implementación de tecnologías para la educación virtual. La penetración del modelo en los sistemas educativos ha sido a través de carreras virtuales que también son prototipos en el ofrecimiento a la población.

\section{Recomendación}

Es necesaria la implementación del modelo en otros sistemas de educación para tener un referente de su aplicabilidad. Se sugiere la continuidad de la investigación usando las redes neuronales como algoritmo de retroalimentación de las reglas difusas de inferencia y el diseño de instrumentos basados en tratamiento inteligente de datos, como el big data, para la programación de un sistema neurodifuso que agilice los cálculos involucrados en el proceso de evaluación objeto de estudio.

Se hace necesario que el sistema de educación promueva el uso masivo de la educación virtual.

\section{Referencias}

Ballester, L. y Colom, A. (2006). Lógica difusa: una nueva epistemología para las ciencias de la educación. Revista de Educación, (340), 995\#1008.

De la Hoz, E., De la Hoz, E. y Herrera, T. (2019). Metodología de Aprendizaje Automático para la Clasificación y Predicción de Usuarios en Ambientes Virtuales de Educación. Información tecnológica. 30(1), 247\#254. https://doi.org/10.4067/S0718-07 642019000100247

García Ferrer, B. (2017). El precio del progreso: de la 'virtualización del mundo' al 'zombismo hiperindividualista'. Recerca, Revista de Pensamiento y Análisis (20), 105\#126. htt ps://doi.org/10.6035/Recerca.2017.20.6

Global Millennial Survey. Telefónica. (2014). Resultados de la encuesta a la generación global del milenio de Telefónica. Los jóvenes de hoy en día son los líderes del mañana. http://www.i nformeticplus.com/global-millennial-survey-2014-telefonica

Ma, J. y Zhou, D. (2000). Fuzzy Set Approach to the Assessment of Student-Centered Learning. IEEE Transactions on Education, (43), 237-241. https://doi.org/10.1109/13.848079

Mendoza, A. A., Ospino, W. A. y Romero, D. (2016). Aplicación de los métodos de toma de decisiones LP\#GW\#AHP y lógica difusa para la selección de una electiva académica en la Universidad del Atlántico, Colombia. Revista Virtual Universidad Católica del Norte, (48), 351\#364.

Ministerio de Educación Nacional. (2018). Referentes de calidad: una propuesta para la evolución del Sistema de Aseguramiento de la Calidad. Mineducación. https://www.mineducacion.gov.c o/1759/articles-369045_recurso.pdf

Shinghal, R. (2013). Introduction to Fuzzy logic. PHI Learning.

Notas

* Texto presentado en el IV Foro Internacional de Investigación en Ambientes Virtuales de Aprendizaje

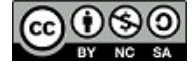

